\title{
Impact of Partial Element Accuracy on PEEC Model Stability
}

\author{
Jonas Ekman, Member, IEEE, Giulio Antonini, Senior Member, IEEE, Antonio Orlandi, Senior Member, IEEE, \\ and Albert E. Ruehli, Life Fellow, IEEE
}

\begin{abstract}
This paper details the impact of partial element accuracy on quasi-static partial element equivalent circuit (PEEC) model stability in the time domain. The potential sources of inaccurate partial element values are found to be poor geometrical meshing and the use of unsuitable partial element calculation routines. The impact on PEEC model stability of erroneous partial element values, and the coefficients of potential and partial inductances, are shown as theoretical constraints and practical results. Projection meshing, which is a discretization strategy suitable for the PEEC method, is shown to improve calculated partial element values for the same number of unknowns, thus improving model stability.
\end{abstract}

\section{Index Terms-Partial element equivalent circuit (PEEC).}

\section{INTRODUCTION}

$\mathbf{T}$ HE need for three-dimensional (3-D) electromagnetic (EM) modeling is increasing due to multi-gigahertz signal bandwidths at all levels of integration and packaging, mixedsignal functionality, and larger wiring densities in complex 3-D environments [1]. In the 2003 International Technology Roadmap for Semiconductors (ITRS) [2], the use of highfrequency 3-D EM modeling is designated as an emerging area. This roadmap states that the need for high-frequency modeling and package electrical modeling will be of increasing importance over the next decade. Solving combined electromagnetic and circuit analysis problems is required for printed circuit board (PCB), subsystem-PCB modeling, and electrical interconnect and package (EIP) problems. Two-dimensional (2-D) multiconductor transmission line (MTL) analysis is used for problems which can be solved in this way. However, where 2-D modeling is inadequate, 3-D modeling techniques must be used.

The partial element equivalent circuit (PEEC) method [3]-[5] is a 3-D full-wave modeling method suitable for combined EM and circuit analysis. Unlike the method of moments (MoM), PEEC is a full spectrum method valid from dc to the maximum frequency determined by the meshing. In the PEEC method, the integral equation is interpreted as Kirchoff's voltage law applied to a basic PEEC cell, which results in a complete circuit solution for 3-D geometries. The equivalent circuit formulation allows for additional SPICE type circuit elements to be easily

\footnotetext{
Manuscript received July 19, 2005; revised October 7, 2005. This work was supported by the Swedish Research Council.

J. Ekman, G. Antonini, and A. Orlandi are with the Department of Electrical Engineering, University of L'Aquila, 67040, L'Aquila, Italy (e-mail: jonas.ekman@ltu.se; antonini@ing.univaq.it; orlandi@ing.univaq.it).

A. E. Ruehli is with the IBM T. J. Watson Research Center, Yorktown Heights, NY 10598 USA (e-mail: ruehli@us.ibm.com).

Digital Object Identifier 10.1109/TEMC.2006.870699
}

included. Further, the models and the analysis apply to both the time-and the frequency-domain. With a general purpose SPICE type solver, different analysis such as quasi-static, LR or RC, and multitransmission line model analysis can be performed. The circuit equations resulting from the PEEC model are easily constructed using a modified loop analysis (MLA) or modified nodal analysis (MNA) formulation [6]. In the MNA formulation, the volume cell currents and the node potentials are solved simultaneously for the discretized structure. This approach is used in most SPICE type circuit solvers. Besides providing a dc solution, it has several other advantages over a MoM analysis for this class of problems since any type of circuit element can be included in a straightforward way with appropriate matrix stamps.

The PEEC method has recently been extended to include nonorthogonal geometries [7]. This model extension, which is consistent with the classical orthogonal formulation, includes the Manhattan representation of the geometries in addition to the more general quadrilateral and hexahedral elements. This helps in keeping the number of unknowns at a minimum and thus reduces computational time for nonorthogonal geometries. The nonorthogonal partial element values are calculated using (semi-)numerical integration over the discretized surfaces and volumes. Thus, there is an increased potential for introducing errors in partial element values compared to the classical rectangular PEEC formulation.

The potential instability of the discretized electric field integral equation (EFIE) in the time domain is well known, e.g., [8]. In this paper, we investigate the time-domain stability of quasistatic PEEC-based EM models from a circuit perspective. We focus on the impact of partial element accuracy on PEEC model stability. In [9], the impact of erroneous coefficients of potential on PEEC model stability was shown. This paper extends the analysis and shows similar results for PEEC model partial inductances. This type of stability analysis is motivated by

1) the application of PEEC modeling for dense and complex systems requiring projection meshing (see Section III-B) to assure correctness of the partial element values [10];

2) the nonorthogonal PEEC formulation [7] which requires the use of numerical space integration rather than analytical formulae in the partial element evaluation;

3) the increased usage of full-wave PEEC solvers for which stability is an important and difficult issue;

4) the use of PEEC modeling for a wide variety of geometries which can result in dense PEEC model submatrices with a wide range of coupling terms, which are prone to numerical errors; 
5) the research on the sparsification of partial inductance matrices [11] by windowing-techniques [12] and wireduplication [13].

This paper is organized as follows. After a short introduction to the PEEC formulation in Section II, PEEC model stability is outlined in Section II-B. In Section III, the different sources contributing to erroneous partial elements are detailed. Section IV describes the stability of PEEC models with respect to partial coefficients of potential. Here we study a symmetrical as well as an asymmetrical PEEC model to show the stability properties. Practical examples are given for stable and unstable quasi-static PEEC models with respect to the derived theoretical conditions. Finally, Section IV-B details the stability of PEEC models with respect to partial inductances. The inductive effects are studied for a two-cell and a transmission line geometry. The same type of stability criteria are derived, and a similar behavior is observed as for the capacitive case. Finally, conclusions are drawn.

\section{BRief Review Over the Partial Element Equivalent CIRCUIT MODEL}

The basic PEEC method is derived from the mixed potential integral equation (MPIE) written as

$$
\boldsymbol{E}^{\mathrm{i}}=\frac{\boldsymbol{J}(\boldsymbol{r}, t)}{\sigma}+\frac{\partial \boldsymbol{A}(\boldsymbol{r}, t)}{\partial t}+\nabla \phi(\boldsymbol{r}, t)
$$

where $\boldsymbol{E}^{\mathrm{i}}$ is an incident electric field, $\boldsymbol{J}$ is the current density, $\boldsymbol{A}$ is the vector magnetic potential, $\phi$ is the scalar electric potential at observation point $\boldsymbol{r}$, and $\sigma$ is the electrical conductivity. By using the definitions of the EM potentials, the current- and charge-densities are discretized by defining pulse basis functions for the conductors and dielectric materials. Pulse functions are also used for the weighting functions, resulting in a Galerkin type solution and the circuit interpretation of the scalar- and vector-potentials. By defining a suitable inner product with a weighted volume integral over the cells, the MPIE in (1) can be interpreted as Kirchhoff's voltage law over a PEEC cell consisting of the following:

1) Partial self inductances between the nodes and partial mutual inductances representing the magnetic field coupling in the equivalent circuit. The partial inductance is defined as

$$
L p_{\alpha \beta}=\frac{\mu}{4 \pi} \frac{1}{a_{\alpha} a_{\beta}} \int_{v_{\alpha}} \int_{v_{\beta}} \frac{1}{\left|\boldsymbol{r}_{\alpha}-\boldsymbol{r}_{\beta}\right|} \cdot d v_{\alpha} \cdot d v_{\beta} .
$$

2) Coefficients of potential to each node and mutual coefficients of potentials between the nodes representing the electric field coupling. The coefficients of potentials are defined as

$$
p_{i j}=\frac{1}{S_{i} S_{j}} \frac{1}{4 \pi \epsilon_{0}} \int_{S_{i}} \int_{S_{j}} \frac{1}{\left|\boldsymbol{r}_{i}-\boldsymbol{r}_{j}\right|} \cdot d S_{j} \cdot d S_{i} .
$$

3) A resistive term between the nodes are defined as

$$
R_{\gamma}=\frac{l_{\gamma}}{a_{\gamma} \sigma_{\gamma}} .
$$

In (2) and (4), $a$ represents the cross section of the volume cell normal to the current direction $\gamma$ and $l$ is the length in the current

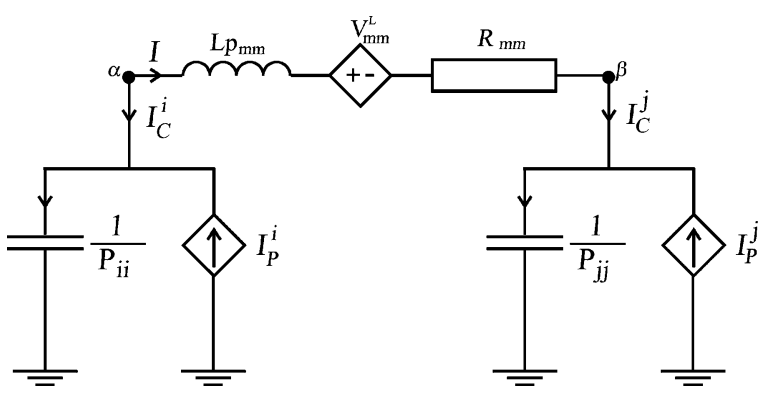

Fig. 1. Basic PEEC cell with conductor loss included.

direction $\gamma$. Further, $v$ represents the current volume cells and $S$ the charge surface cells. The final PEEC cell can be constructed in different ways depending on the required type of analysis. Fig. 1 shows one basic circuit which is most suitable for use with the MNA method. In Fig. 1, a retarded current controlled voltage source (CCVS), $V_{m m}^{L}$ is used to model retarded magnetic field coupling while retarded CCCSs $I_{p}^{i}$ and $I_{p}^{j}$ model the electric field couplings for a PEEC cell connected to nodes $\alpha$ and $\beta$. For example, the value for $I_{p}^{i}=\left(\left(p_{i j}\right) /\left(p_{i i}\right)\right) I_{C}^{j}\left(t-t_{d_{i j}}\right)$, where $t_{d_{i j}}$ is the center-to-center retardation time given by

$$
t_{d_{i j}}=\frac{\left|\boldsymbol{r}_{\mathrm{c} i}-\boldsymbol{r}_{\mathrm{c} j}\right|}{c}
$$

where $\boldsymbol{r}_{\mathrm{c} i}$ and $\boldsymbol{r}_{\mathrm{c} j}$ are the vectors to the center of the surface cells $i$ and $j$, respectively. Further, the free space speed of light $c=3 \cdot 10^{8} \mathrm{~m} \cdot \mathrm{s}^{-1}$ is used for combined conductor and dielectric material problems due to the transformation to a free space problem using a volumetric equivalence principle. The transformation of the EM problem to the circuit domain is thereby completed and the total PEEC can be solved using circuit techniques. For a detailed derivation of the method which includes the nonorthogonal formulation, see [7]. Importantly, the nonorthogonal formulation does not change the topology or basic properties of the PEEC circuit model. Hence, the work in this paper applies to the general case.

\section{A. Meshing for the PEEC Model}

An important part of PEEC modeling is the meshing of the structure. In the method, two discretizations are constructed. After the initial node placement, the surfaces are meshed using quadrilateral elements from which the coefficients of potentials are calculated using (3). Depending on the boundaries of the surface mesh, the volume cells are created as hexahedral cells from which the partial inductances and cell resistances are calculated using (2) and (4), respectively. The usage of quadrilateral and hexahedral elements in the meshing gives a very good geometric meshing. However, the use of rectangular surface-cell and parallelepiped volume-cell in the meshing, which gives a staircase approximation of the structure, enables the use of fast and accurate analytical calculation routines for the partial elements. The possibility to combine the nonorthogonal and orthogonal shapes should be used as much as possible to speed up the partial element calculation phase.

The basic rule of thumb when performing the surface-and volume-cell discretization is to use a fixed number of cells 


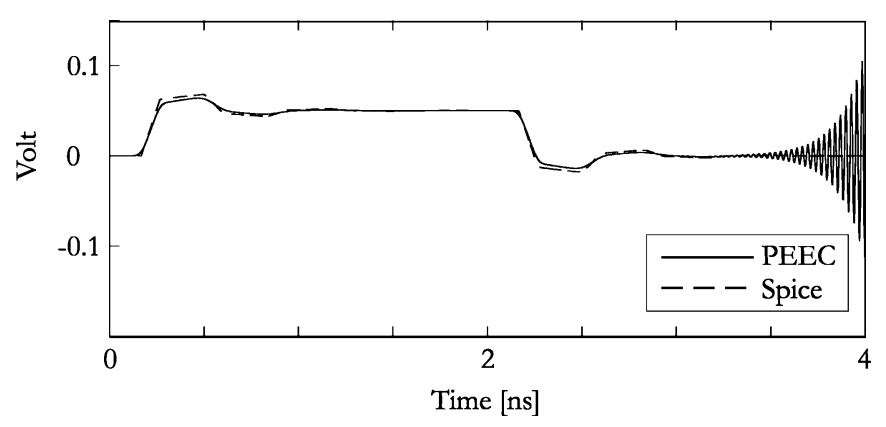

Fig. 2. Short time step late time instability for a PEEC model. Example compares far end voltage for a transmission line modeled using an unstable PEEC and a SPICE model.

per shortest wavelength $\lambda_{\min }$ (corresponding to the highest frequency of interest) to assure a correct representation of the actual waveforms. Originally, approximately (10 cells $\left./ \lambda_{\min }\right)$ was used. This has changed to $\left(20\right.$ cells $\left./ \lambda_{\min }\right)$ for current PEEC models. Further, conditions determining the level of the discretization, i.e., the sizes of the volume- and surface-cells, are determined by

1) The shape of the conductors. Length to width and length to thickness ratios must be considered to ensure good accuracy in the calculated partial element values [14]. Too large ratios in the cell dimensions can cause numerical problems in the partial element calculation routines when using numerical integration and closed-form equations.

2) The proximity to other conductors. For closely spaced, overlapping, parallel, or perpendicular conductors, the discretization in some cases has to be commensurate.

3) Time and memory limitations. The number of partial elements used in a PEEC model is linked to the discretization. Thus, the calculation time of the partial elements and the solution time of the final system increases drastically for overdiscretized problems.

\section{B. Time-Domain PEEC Model Stability and Accuracy}

The time-domain stability issue for PEEC models and other full-wave integral equation based formulated EM analysis methods is well known, e.g. [8]. The problem that is often termed "late time instability" results in an oscillation of an exponentially increasing amplitude which starts at some point in time while totally masking the real solution, as shown in Fig. 2, which may occur even for implicit numerical integration schemes.

Reasons for PEEC model time-domain instabilities are

1) delays in full-wave formulations;

2) properties of some explicit and implicit numerical timedomain integration technique [8], [15], [16];

3) poor meshing of geometry (which is also related to the delay discretization for full-wave models, e.g., [17]).

These issues have been treated in the literature and solutions have been proposed to improve the stability of PEEC models based on these analyses. In this paper, we consider a fourth issue which impacts PEEC model stability, i.e., the accuracy of the partial element values, both coefficients of potential and partial inductances. It will be shown in the following sections that the factors contributing to inaccuracies in partial element calculations are

1) inaccurate partial coefficient calculations;

2) the use of large aspect ratio PEEC cells for which certain calculation routines perform poorly;

3) the inappropriate discretization of objects without proper adaptive meshing routines.

The consequences of inaccurately calculated partial elements in the final PEEC model solution are hard to predict. However, an attempt is made in this paper to illustrate the possibility that the errors will be emphasized when creating the PEEC circuit equations and the required matrix operations. Fig. 3 shows a flow chart for a general PEEC-based EM analysis tool with emphasis on approximations and error sources which can result in an unstable PEEC model. Here, we focus on the errors introduced in partial element calculation and the numerical errors introduced by the matrix operations.

\section{SOURCES OF ERroneous PARTIAL ElEMENT EVAluATION}

In the PEEC method, a large number of partial inductances and coefficients of potentials must be calculated which are used in the equivalent circuit. The partial elements are calculated depending on the surface- and volume-cell discretization, which is based on the highest frequency $f_{\max }$ the PEEC model is intended for so that the cell size does not exceed the $\left(\lambda_{\min } / 20\right)$ rule, where $\lambda_{\min }=c / f_{\max }$. The close proximity to other conductors/dielectrics and the shape of the conductors may cause problems. For the classical (orthogonal) PEEC formulation, the partial elements are calculated using fast and accurate analytical formulae while nonorthogonal partial elements require at least some numerical (space) integration. The following sections show possible errors introduced in partial elements by calculation routines and by unsuitable surface-and volume-meshing.

\section{A. Partial Element Calculation Routines}

This section addresses the importance of using appropriate techniques when calculating the partial elements. Examples of unstable and unsuitable routines are given for both the analytical and numerical routines.

1) Analytical Formulae: The use of analytical formulae for partial element calculation in PEEC modeling is desirable due to fast and simple usage, especially for the self-terms. For orthogonal self-elements, the analytical formulae are unconditionally used due to the singularity in the integrand. However, even analytical formulae have to be used with care. One of the benefits of PEEC modeling is the possibility to use large-aspect-ratio cells in the discretization. This requires the use of routines so that numerical inaccuracies, with large aspect ratios as illustrated in Fig. 4, can be avoided. Fig. 4 gives an example of how an analytical formula used to calculate the partial self-inductance for a PEEC model volume cell can produce totally wrong inductance values, including even negative results for the partial self-inductance. It was shown in [3] and [18] that the major source of error in partial inductance calculations is due to largeaspect-ratio cells and the result of the numerical dispersion due to high-order terms creating large numbers while the values 


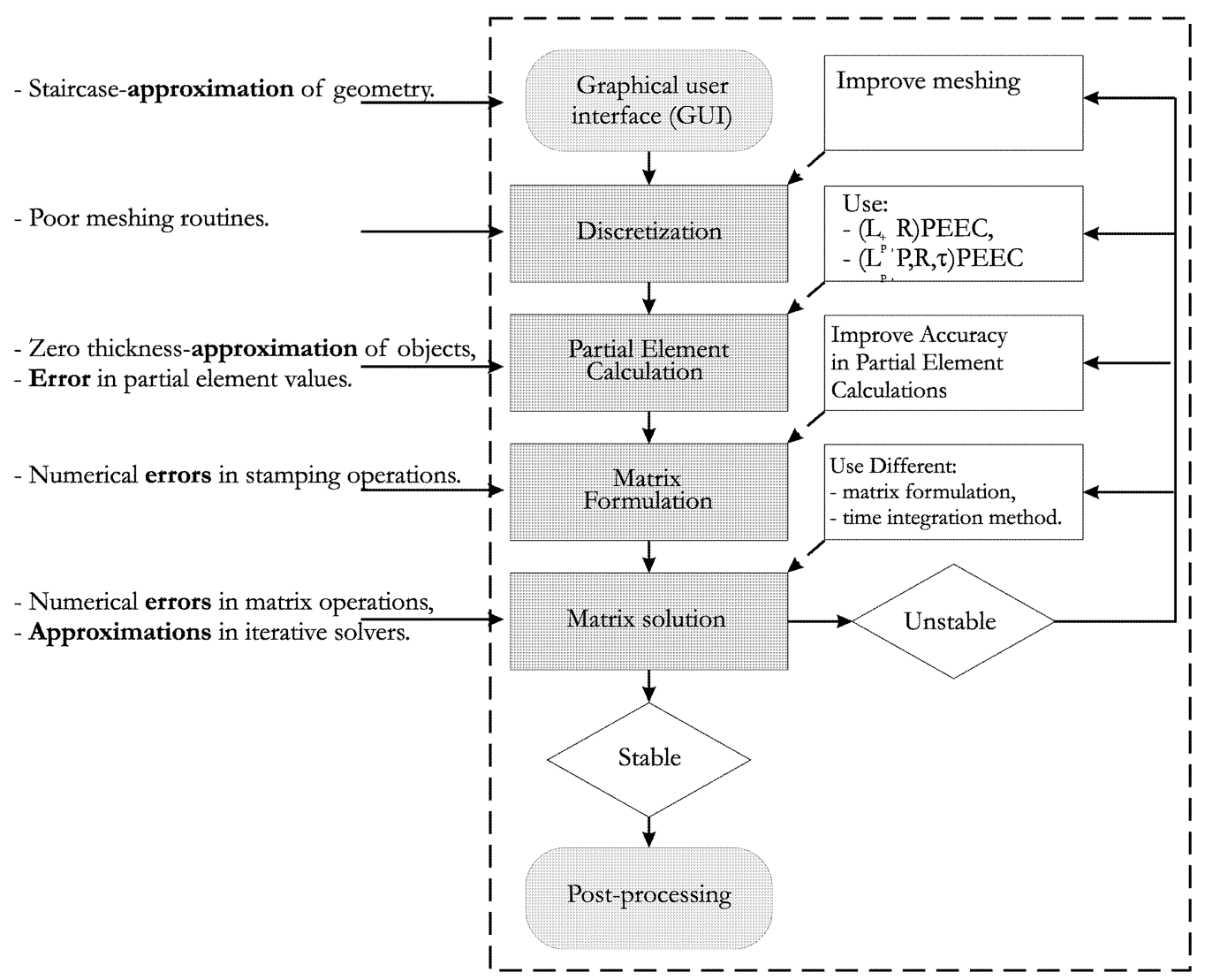

Fig. 3. Flowchart describing a general PEEC based EM simulation tool.

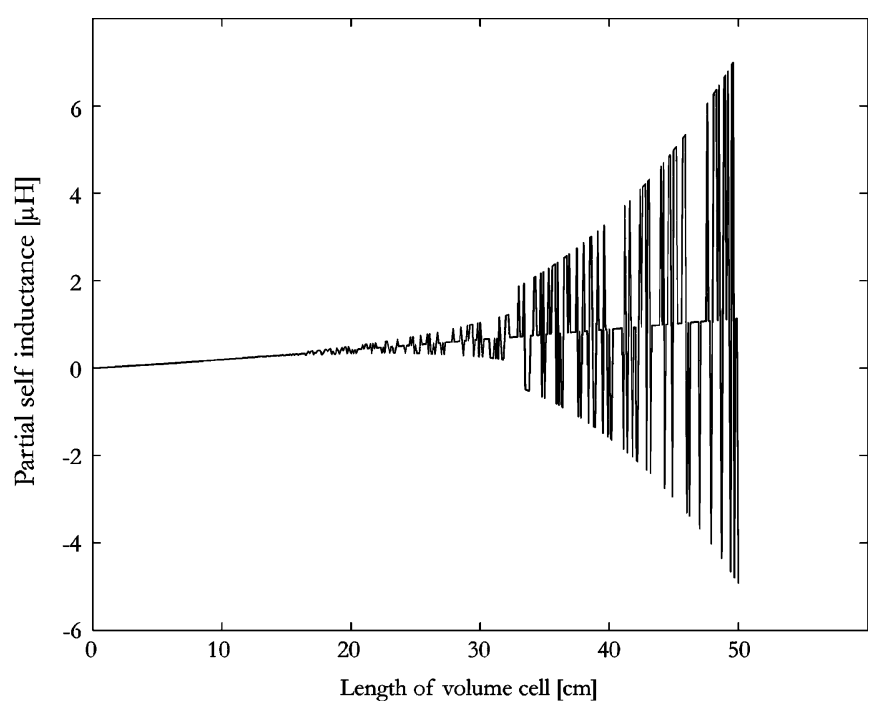

Fig. 4. Numerical unstable partial self-inductance calculation routine; constant cross section $(0.1 \times 0.1 \mathrm{~mm})$ with increasing length.

of other terms are small. Thus, the implementation of self and mutual partial inductance formulae has to be done with care and some size limitations must be specified.

Previously published guidelines [14] using conditioning based on cell length, width, and thickness have proven to be valuable when choosing analytical formulae for the partial coefficient evaluation.
2) Numerical Integration: Numerical integration for the evaluation of the basic partial element expressions is used for nonorthogonal structures or when analytical formulae are difficult to find. The common use of large-aspect-ratio cells and/or large separation distances puts difficult constraints on the numerical integration routines. Hence, an adaptive strategy must be used to accommodate wide variations in the coefficients [19]. Further, the wide range of approximations for the basic partial element expressions, surface, and contour-formulations, can help from an efficiency point of view.

Fig. 5 shows the calculated partial self-inductance $L p$ for an orthogonal cell with $0.1 \times 0.1 \mathrm{~mm}$ cross section $(w \times h)$ with increasing length ( $x$-axis) using the scheme proposed in [14] by means of analytical functions compared to a 3-D (volume) formulation using fifth or eighth order of GaussLegendre (GL) numerical integration [20]. The figure also shows the relative error, which is defined as err $=\left(L p_{\text {numerical }}-\right.$ $\left.L p_{\text {analytic }} / L p_{\text {analytic }}\right)$ in the numerical calculation routines. It is clear that even the eighth order of Gauss-Legendre numerical integration gives poor results for conductor aspect ratios $l: w$ of $2: 1$ since the relative error in the order of $1.5 \%$ is much too large for dense problems. Also, a very careful implementation is required to avoid large compute time when using even higher order GL numerical integration techniques.

As an example, the result for the partial mutual inductance for two center-aligned cells is shown in Fig. 6. The calculation routines are LpFilNum [volume filament model (VFI) formula using 

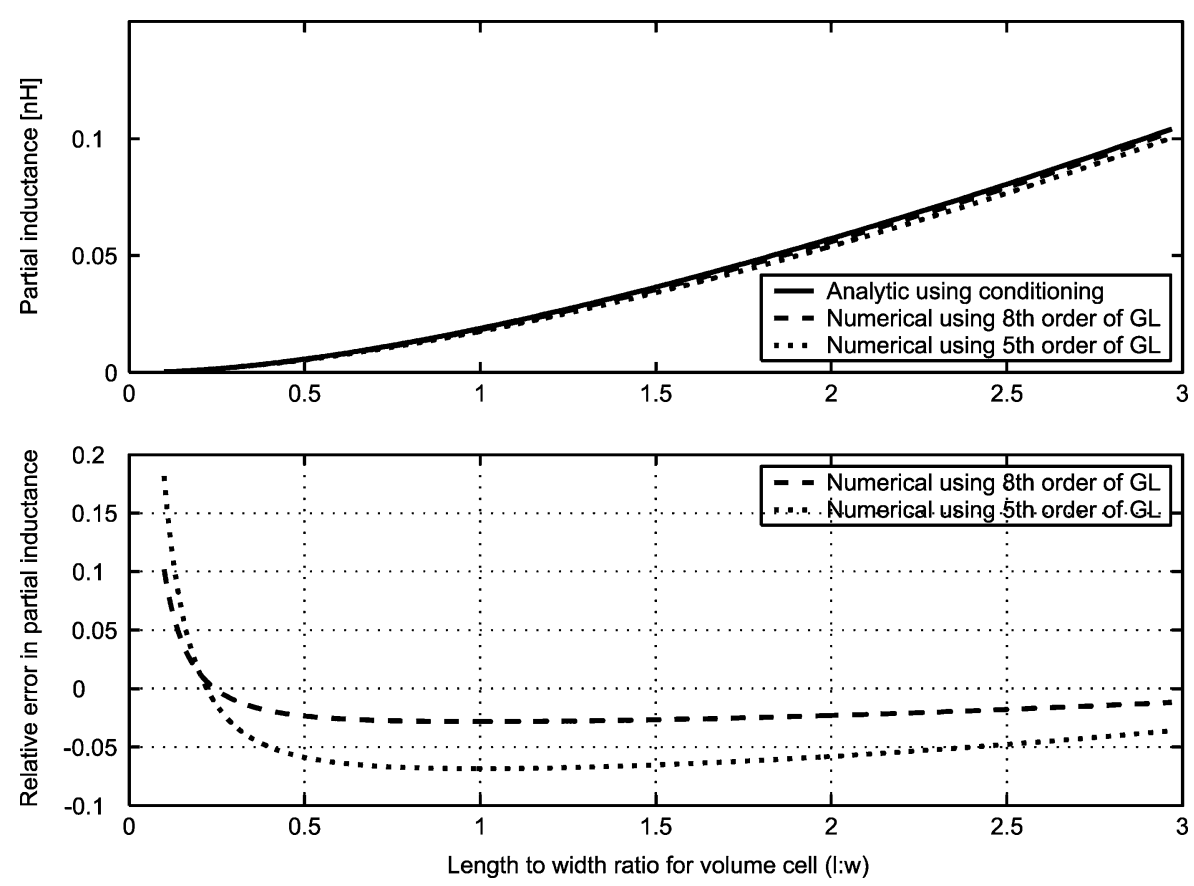

Fig. 5. Comparison for partial self-inductance calculation using analytical (exact) or numerical (volume) techniques and corresponding relative error for finite thickness conductors.

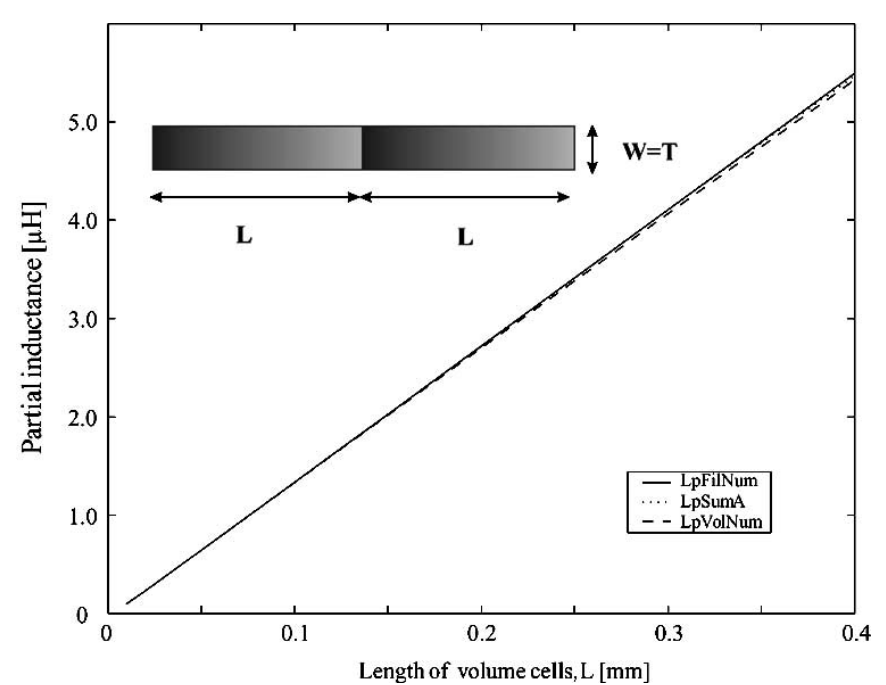

Fig. 6. Partial mutual inductance for two orthogonal cells with increasing cell lengths.

GL numerical integration], LpVolNum (volume formulation us-

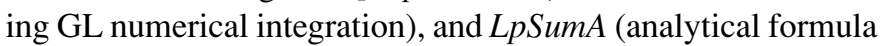
using the summation of 64 partial self-inductances [21]). As can be seen, the result is similar to the previous performed partial self-inductance calculation. Both the volume formulation and the analytical formula ( $\operatorname{LSSumA}$ ) underestimate the partial mutual inductance compared to the filament formula.

We experimentally found the following.

1) Using the volume formulation with low order of GaussLegendre numerical integration can underestimate the partial self-inductance of a rectangular conductor unless aspect ratio is less than 0.25 for finite thickness conductors.
2) The contour formulation [22] slightly overestimates the partial self-inductance of a zero-thickness conductor.

The consequences of over- or under-estimating the partial inductances can be an important source of instabilities when mixing analytical and numerical-based calculation routines for large problems of combined orthogonal and nonorthogonal objects. Both over- and under-estimating partial self-elements leads to problems since the partial inductance matrix $\mathbf{L}_{p}$ must be positive definite. For example, underestimating self terms can result in mutual terms that are larger and thus create an indefinite $\mathbf{L}_{p}$-matrix, as shown below.

This section has illustrated the potential error in partial inductance calculations using different available formulations. It is evident that care must be taken in the evaluation of partial elements.

The partial elements are best calculated using schemes of the type presented in [14] and [19]. When numerical integration is used, the integration order for large-aspect-ratio cells $(1: 100)$ may need to be too high.

\section{B. Impact of Meshing on Partial Elements}

Meshing is another key issue in the accurate and stable modeling. One of the objectives of the meshing in the PEEC approach is the careful allocation of the unknowns or subdivisions. A technique which is called projection meshing is used to obtain convergent results without an excessive number of cells. In this approach, cells for neighboring conductors are lined up, as in the example in Fig. 7. It was shown in [10] that capacitive couplings could be completely wrong if projection meshing was not used for close conductors. It is obvious from this example that the proper meshing for multiple conductor geometries is very important. 


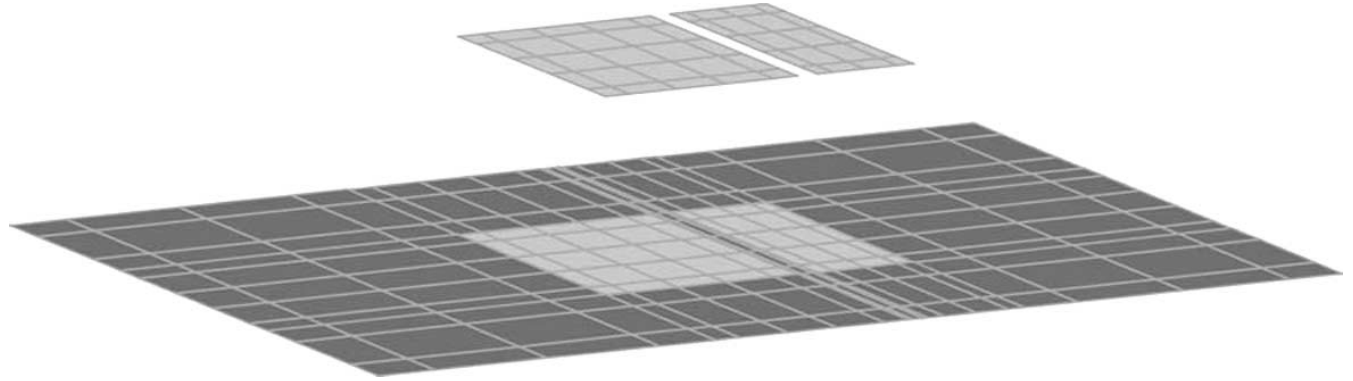

Fig. 7. Projection meshing for a planar inverted F antenna (PIFA) PEEC model. The surface cell discretization of the two antenna elements is mirrored in the ground plane.

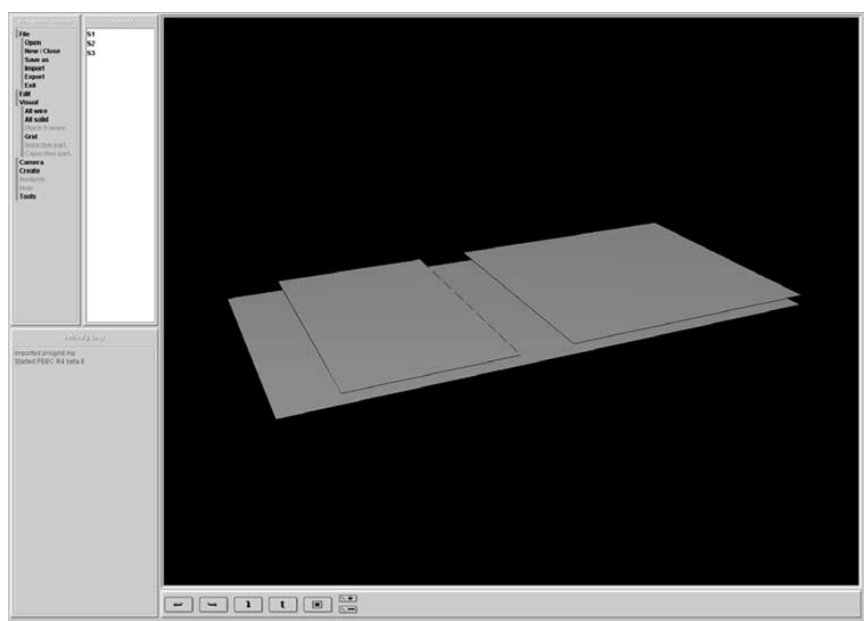

Fig. 8. Three-conductor geometry.

In this section, we give an example similar to the one in [10] to display the importance of projection meshing on the partial elements. Consider the three, zero-thickness, conductor geometry in Fig. 8. The lower plate (No. 1) is $10 \times 5 \mathrm{~cm}$, the upper left plate (No. 2) is $3 \times 5 \mathrm{~cm}$, and the upper right plate (No. 3) is $5 \times 5 \mathrm{~cm}$. The gap separation between the upper plates (Nos. 2 and 3) is $1 \mathrm{~cm}$, and the interplane separation is $0.2 \mathrm{~cm}$.

The capacitive coupling between the plates is given by the short circuit capacitance matrix $\mathbf{C}_{\mathrm{S}}$ as detailed in Section IV-A. To find $\mathbf{C}_{S}$, we analytically compute the potential coefficients [4]. In the first test, we use the smallest number of unknowns, which is three. For this case, the matrix of potential coefficients is given by

$$
\mathbf{P}=\left[\begin{array}{lll}
0.367179683 & 0.355179322 & 0.346424918 \\
0.355179322 & 0.679185447 & 0.185787332 \\
0.346424918 & 0.185787332 & 0.534448842
\end{array}\right]\left[\mathrm{pF}^{-1}\right]
$$

(6) which results in the following short circuit capacitance matrix:

$$
\mathbf{C}_{\mathrm{S}}=\left[\begin{array}{ccc}
18.88094007 & -7.21178058 & -9.73146582 \\
-7.21178058 & 4.38168922 & 3.15143006 \\
-9.73146582 & 3.15143006 & 7.08342159
\end{array}\right][\mathrm{pF}] .
$$

This result is clearly wrong since this $\mathbf{C}_{\mathrm{S}}$ matrix is supposed to be a positive definite Minkowski M-matrix with all its off diagonals being negative. Obviously, the above discretization is very coarse and gives inaccurate results for the short circuit capacitance matrix. To overcome this problem, the lower plate can be subdivided, as an example, into four parts. In a first test, the lower plate is divided into four parts where No. $1_{1}$ is $2.5 \times 5 \mathrm{~cm}$, No. $1_{2}$ is $3.5 \times 5 \mathrm{~cm}$, No. $1_{3}$ is $2.5 \times 5 \mathrm{~cm}$, and the last part No. $1_{4}$ is $1.5 \times 5 \mathrm{~cm}$, as shown in Fig. 9(a). Based on the new discretization, the matrix of potential coefficients is given in (8), shown at the bottom of the page, which results in a short circuit capacitance matrix, after reduction as detailed in [23], given by

$$
\mathbf{C}_{\mathrm{S}}=\left[\begin{array}{ccc}
20.5104891 & -6.8947201 & -11.7813246 \\
-6.8947201 & 6.2414666 & 0.94317315 \\
-11.781325 & 0.9431732 & 11.55098574
\end{array}\right][\mathrm{pF}] .
$$

The resultant $\mathbf{C}_{\mathrm{S}}$ matrix is still erroneous, due to the positive off diagonal terms, even for this increased discretization. In a second test, the lower plate (No. 1) is subdivided into four parts, where No. $1_{1}$ is $1 \times 5 \mathrm{~cm}$, No. $1_{2}$ is $3 \times 5 \mathrm{~cm}$, No. $1_{3}$ is $1 \times 5 \mathrm{~cm}$, and the last part (No. $1_{4}$ ) is $5 \times 5 \mathrm{~cm}$, which projects the shape of the two upper plates; see Fig. 9(b). This case resembles the projection meshing, and this allows for the capacitive couplings to be calculated much more accurately, and the resulting coefficient of potential matrix is shown in (10) at the bottom of the next page. This results in a short circuit capacitance matrix,

$$
\mathbf{P}=\left[\begin{array}{cccccc}
0.7343593661 & 0.295629091 & 0.146034639 & 0.110132751 & 0.376796726 & 0.145632455 \\
0.2956290912 & 0.633887687 & 0.295629091 & 0.174569307 & 0.558728794 & 0.308104658 \\
0.1460346388 & 0.295629091 & 0.734359366 & 0.390004672 & 0.229968963 & 0.52803377 \\
0.1101327506 & 0.174569307 & 0.390004672 & 0.896396661 & 0.151562625 & 0.467811551 \\
0.3767967255 & 0.558728794 & 0.229968963 & 0.151562625 & 0.679195447 & 0.240297211 \\
0.1456324546 & 0.308104658 & 0.528033767 & 0.467811551 & 0.240297211 & 0.534448842]
\end{array}\right]\left[\mathrm{pF}^{-1}\right]
$$




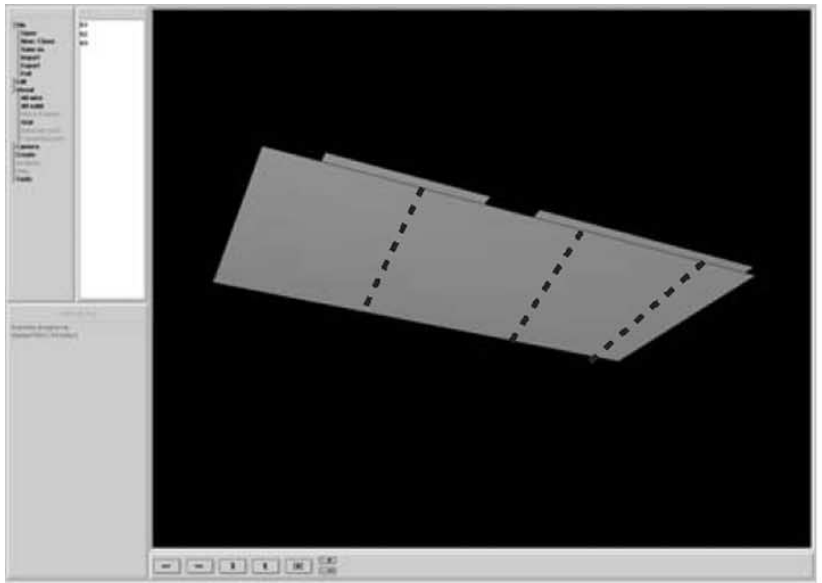

(a)

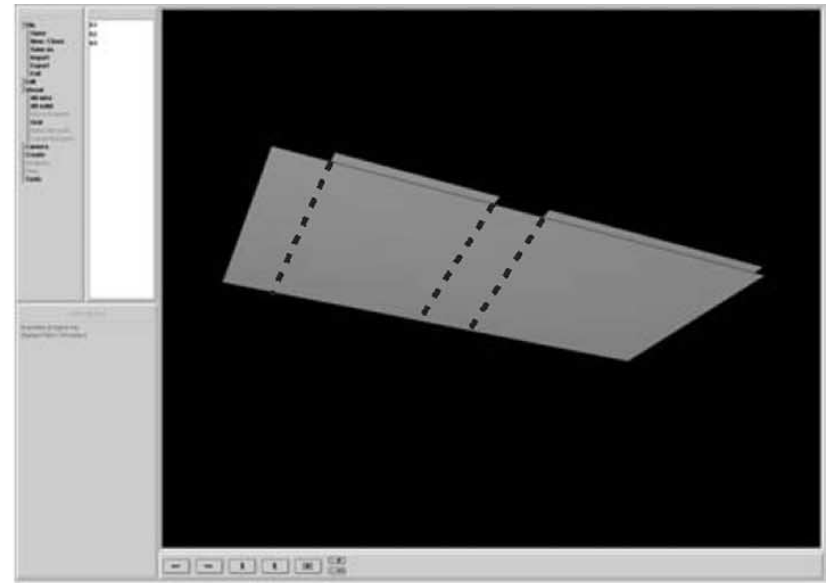

(b)

Fig. 9. Bottom view of three conductor geometry from Fig. 8 with lower plate subdivided, indicated by dashed lines, into (a) four arbitrary areas and (b) four areas which project the shape of the two upper plates.

after reduction, given by

$$
\mathbf{C}_{\mathrm{S}}=\left[\begin{array}{ccc}
21.3179316 & -7.6205393 & -12.0063976 \\
-7.6205393 & 8.1683522 & -0.1459876 \\
-12.0063976 & -0.1459876 & 12.8934069
\end{array}\right][\mathrm{pF}] .
$$

This simple example shows that poor meshing introduces error in the partial element values if we do not use a projection meshing-type approach, where neighboring cells are matched in the limit as the distance between the conductors goes to zero.

In the next two sections, the impact of inaccurate partial elements and the passivity and stability of simple PEEC models are investigated.

\section{IMPACT OF INACCURATE PARTIAL ELEMENTS ON PEEC MODELS}

\section{A. Capacitive Coupling}

The capacitive effects in PEEC are modeled by using the theory of partial coefficients of potential $p_{i j}$ calculated using the basic expression in (3). In the PEEC method, a coefficient of potential matrix $\mathbf{P}$ is constructed with elements of the type $p_{i j}$, which may be more dense than the partial inductance matrix for rectangular geometries. For PEEC, the $\mathbf{P}$ matrix is full, symmetric, and positive definite [4]. The energy for the potential coefficient can be written as a quadratic form, or

$$
W_{\mathrm{e}}=\frac{1}{2} \mathbf{Q}^{T} \mathbf{P Q}
$$

where $\mathbf{Q}$ is the vector of total cell surface charge. It is worth noticing that all elements in $\mathbf{P}$ are positive as is evident from (3). For a quasi-static PEEC model, lumped capacitances can be used from the inverse of the $\mathbf{P}$ matrix or

$$
\mathbf{C}_{\mathrm{S}}=\mathbf{P}^{-1}
$$

where $\mathbf{C}_{\mathrm{S}}$ is a short circuit capacitance M-matrix with positive diagonal and negative off-diagonal elements. Hence, it is evident that any positive off-diagonal element in the $\mathbf{C}_{S}$-matrix indicates an error in the analysis which may be caused by meshing or a combination of strong and weak couplings, as well as numerical errors in the coefficients. The circuit two-terminal capacitances are obtained by manipulating the $\mathbf{C}_{\mathrm{S}}$ matrix according to the following:

1) The diagonal terms are obtained by summing each row in the $\mathbf{C}_{\mathrm{S}}$-matrix, $c_{i i}=\sum_{\forall j} c_{s_{i j}}$.

2) The off-diagonal terms are the negative value for the corresponding off-diagonal element in the $\mathbf{C}_{S}$-matrix, i.e., $c_{i j}=-c_{s_{i j}}$.

Thus, it is evident that all two-terminal circuit capacitances should be positive.

Here, the stability of a lossless quasi-static PEEC model can be represented in terms of circuit capacitances. A simple onecell PEEC circuit is considered in Fig. 10. It is well known from circuit theory that lumped circuits without independent as well as dependent sources and with positive circuit elements are passive. We use the single cell model to illustrate the impact of inaccurate potential coefficients on stability. To monitor the stability and passivity of the circuit, we study the input impedance at the point marked $Z_{\mathrm{IN}}$ in Fig. 10. It is evident that both nodes

$$
\mathbf{P}=\left[\begin{array}{llllll}
1.030925839 & 0.397998118 & 0.205316464 & 0.128567385 & 0.392105994 & 0.128507242 \\
0.397998118 & 0.679195447 & 0.397998118 & 0.185787332 & 0.613305887 & 0.185532714 \\
0.205316464 & 0.397998118 & 1.030925839 & 0.313623242 & 0.392105994 & 0.310018101 \\
0.128567385 & 0.185787332 & 0.313623242 & 0.534448842 & 0.185532714 & 0.493825139 \\
0.392105994 & 0.613305887 & 0.392105994 & 0.185532714 & 0.679195447 & 0.185787332 \\
0.128507242 & 0.185532714 & 0.310018101 & 0.493825139 & 0.185787332 & 0.534448842
\end{array}\right]\left[\mathrm{pF}^{-1}\right] .
$$




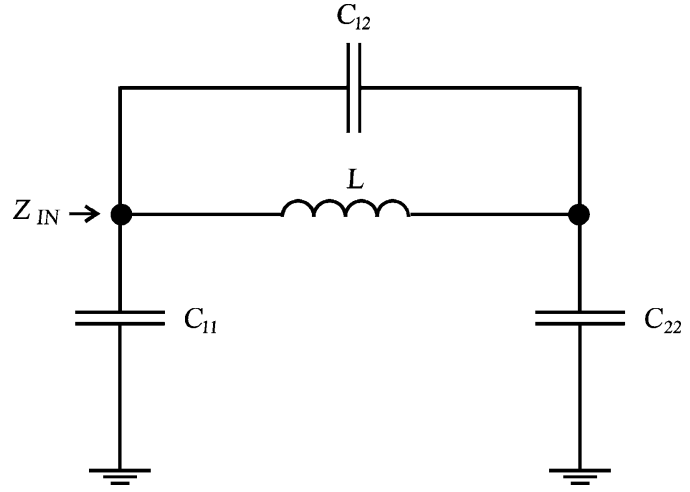

Fig. 10. Simple PEEC circuit used for quasi-static EM problems.

in the small circuit are observable from this point. Stability can be checked by using the condition that all eigenvalues of the system are in the left half plane; see [24]. The input impedance for the symmetric $\left(C_{11}=C_{22}\right)$ one-cell model is given by

$$
Z_{\mathrm{IN}}=\frac{s^{2} L C_{11}+s^{2} L C_{12}+1}{s\left(s^{2} L\left(C_{11}^{2}+2 C_{11} C_{12}\right)+2 C_{11}\right)}
$$

with stability condition

$$
C_{12}>-\frac{C_{11}}{2}
$$

Further, the input impedance for an asymmetric subdivided PEEC one-cell $\left(C_{11} \neq C_{22}\right)$ is shown in (16) at the bottom of the page, with stability condition

$$
C_{12}>-\frac{C_{11} C_{22}}{C_{11}+C_{22}} \text {. }
$$

If the above condition is not fulfilled, the one-cell system can have poles of the transfer function $Z_{\mathrm{IN}}$ with positive real parts causing the system to be unstable. However, for this case, passivity is not always ensured by satisfying (17) since negative values of $C_{12}$ are allowed.

The next section explores the practical implications of the stability criterion developed above by modeling the one-cell in PSpice [25] for a few different cases.

1) Numerical Experiments: First a symmetric one-cell geometry is considered. The length is $5 \mathrm{~cm}$, the width is $2 \mathrm{~cm}$, and the thickness is $1 \mu \mathrm{m}$ and is assumed to be zero thickness in the calculations. The PEEC model matrices are as follows:

$$
\begin{aligned}
\mathbf{P} & =\left[\begin{array}{cc}
1.19146 & 0.41638 \\
0.41638 & 1.19146
\end{array}\right]\left[\mathrm{pF}^{-1}\right] \\
\mathbf{C}_{\mathrm{S}} & =\left[\begin{array}{cc}
0.956 & -0.334 \\
-0.334 & 0.956
\end{array}\right][\mathrm{pF}]
\end{aligned}
$$

which results in two-terminal capacitances according to $C_{11}=$ $C_{22}=0.622 \mathrm{pF}$ and $C_{12}=C_{21}=0.334 \mathrm{pF}$. The partial selfinductance is $\left(L_{p}\right) 22.36 \mathrm{nH}$. The one-cell is excited using a current source pulse with $1 \mathrm{~ns}$ rise time at the node indicated with $Z_{\mathrm{IN}}$ in Fig. 10. Figs. 11 and 12 show the responses, calculated by PSpice, for different mutual capacitance $C_{12}$ values. In the figures, the near end voltage is indicated $V$ in SPICE and the far end voltage is indicated $V$ out SPICE. In Fig. 11, the voltages for the symmetric one-cell with both correct and a negative mutual capacitance are shown. When the capacitance is given a value $-0.32 \mathrm{pF}$, violating the condition in (15), the model is unstable (Fig. 12). These results are consistent with the theoretical constraints developed earlier.

We next consider the one-cell with an asymmetric geometrical discretization where the coefficients of potentials are calculated from two different sized cells. As an example, we choose 4.75 and $0.25 \mathrm{~cm}$ for the two lengths, resulting in the following PEEC model matrix:

$$
\mathbf{P}=\left[\begin{array}{ll}
0.82921 & 0.50647 \\
0.50647 & 2.97818
\end{array}\right]\left[\mathrm{pF}^{-1}\right]
$$

resulting in the following two-terminal capacitances: $C_{11}=$ $0.112 \mathrm{pF}, C_{12}=C_{21}=0.023 \mathrm{pF}$, and $C_{22}=0.015 \mathrm{pF}$. The self partial inductance $\left(L_{p}\right)$ is $22.36 \mathrm{nH}$ as before. The onecell is excited using the same current source as before, resulting in the responses in Figs. 13 and 14. The same behavior as for the symmetric one-cell is observed with a stable response (see Fig. 13) for a mutual capacitance fulfilling the condition in (17) while an unstable response is given for a capacitance value equal of $-0.014 \mathrm{pF}$ [violating condition (17)] (see Fig. 14).

The results are in accordance with the theoretical constraints developed earlier. Importantly, we conclude that the asymmetric discretization puts a stronger constraint on the accuracy in the mutual capacitance term $C_{12}$. This corresponds to realistic models since the cell size varies widely for practical problems and it is consistent with the practical observation that unequal cells lead to more stability problems.

2) $\left(L_{p}, P\right)$ PEEC Model Analysis: The previously analyzed one-cell can also be simulated using a PEEC-based EM solver using the original PEEC model matrices. In this way, constraints on the coefficients of potential, instead of the short circuit capacitances, can be derived and verified. Consider again the symmetric one-cell from the previous section. By using the PEEC based EM solver, the one-cell model matrices are calculated, and a system is created using a nodal analysis (NA) method. The one-cell input impedance is

$$
\mathbf{Z}_{\mathrm{IN}}=\left(\left(\mathbf{A}^{T}(j \omega \mathbf{L})^{-1} \mathbf{A}+j \omega\left(\mathbf{R}_{\mathbf{v}}^{T} \mathbf{P}^{-1} \mathbf{R}_{\mathbf{v}}\right)\right)^{-1} \mathbf{I}_{S}\right)\left[\begin{array}{ll}
1 & 0
\end{array}\right]^{T}
$$

when using the NA formulation, with corresponding matrices, detailed in [26] or when computed

$$
\mathbf{Z}_{\mathrm{IN}}=\frac{s^{2} L_{p} P_{11}+P_{11}^{2}-P_{12}^{2}}{s\left(s^{2} L_{p}+2 P_{11}-2 P_{12}\right)}
$$

$$
Z_{\mathrm{IN}}=\frac{s^{2} L C_{22}+s^{2} L C_{12}+1}{s\left(s^{2} L C_{22} C_{11}+s^{2} L C_{12} C_{11}+s^{2} L C_{12} C_{22}+C_{11}+C_{22}\right)}
$$




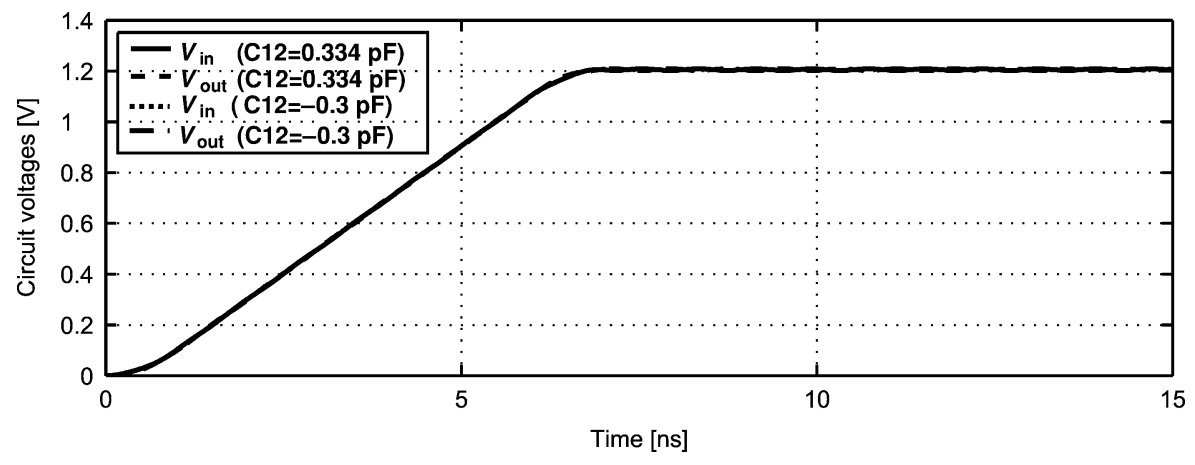

Fig. 11. Stable responses for symmetric one-cell with $C_{12}=0.334 \mathrm{pF}$ and $C_{12}=-0.3 \mathrm{pF}$.

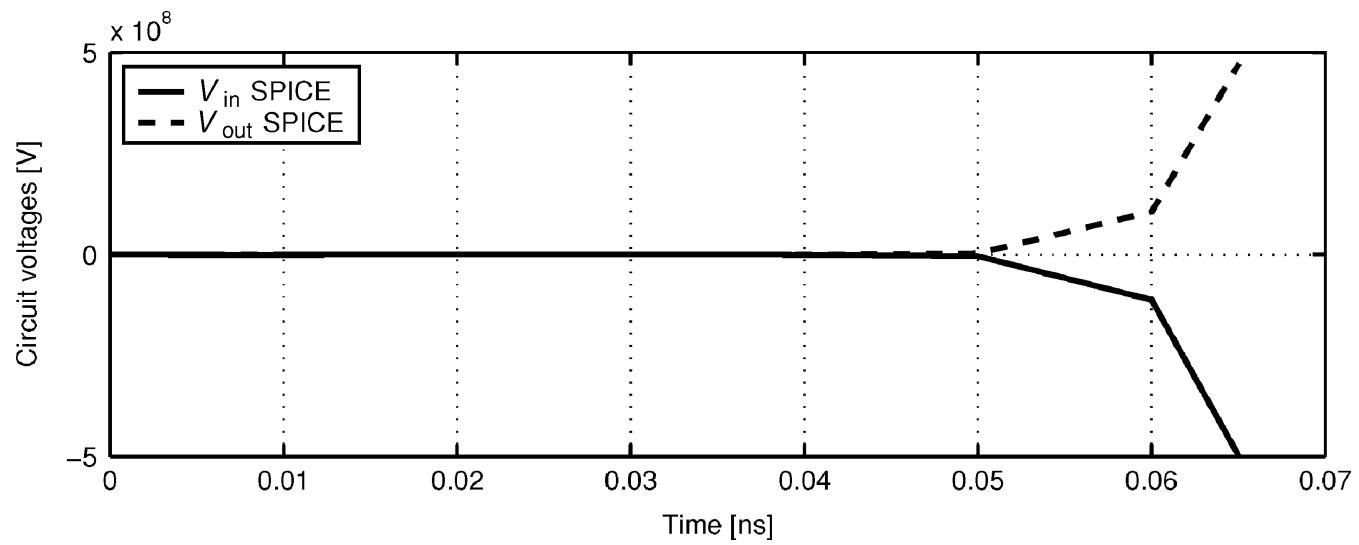

Fig. 12. Unstable response for symmetric one-cell with $C_{12}=-0.32 \mathrm{pF}$, violating stability condition in (15).

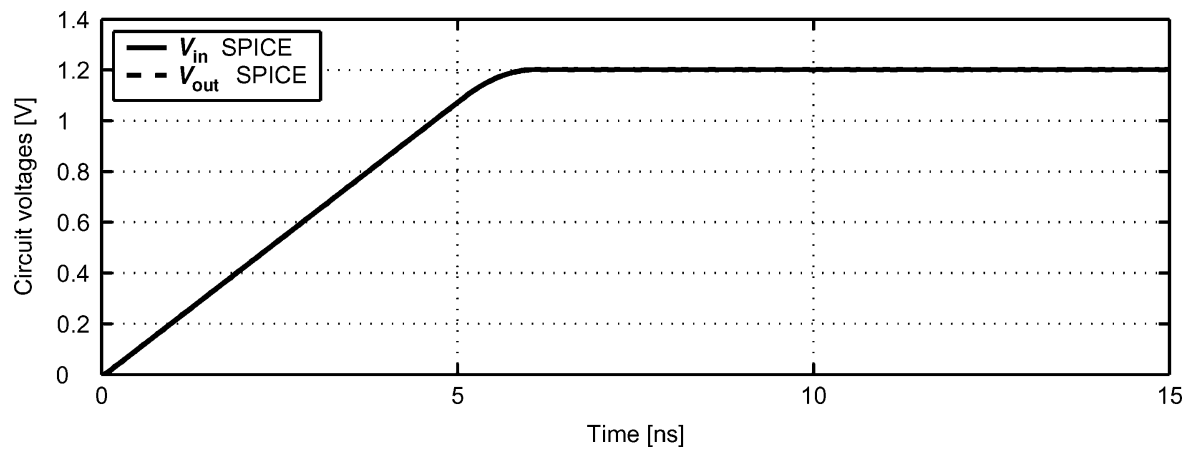

Fig. 13. Stable response for asymmetric one-cell with $C_{12}=0.023 \mathrm{pF}$.

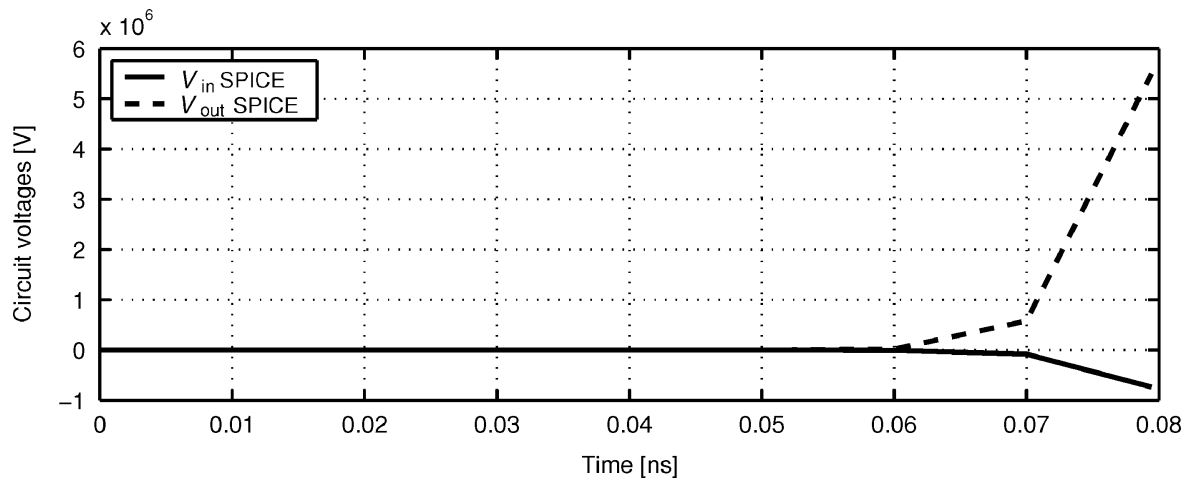

Fig. 14. Unstable response for asymmetric one-cell with $C_{12}=-0.01414 \mathrm{pF}$, violating stability condition in (17). 


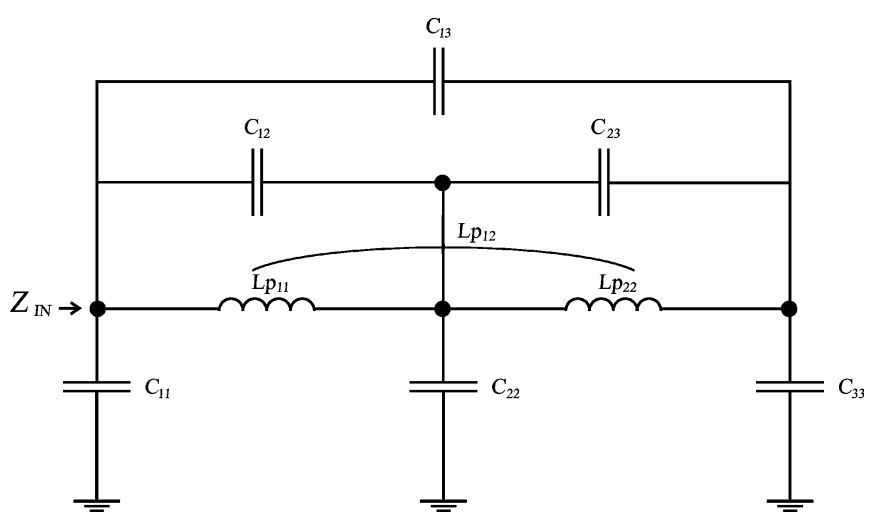

Fig. 15. Quasi-static PEEC two-cell, $L p_{12}$ indicate the partial mutual inductance.

with stability criterion, previously calculated as

$$
P_{12}<P_{11}
$$

The condition in (23) is equal to one of the criteria for positive (semi)definite matrices and can be converted into (15) and (17) by using the coefficient of potential matrix expressed in terms of circuit capacitances according to

$$
\mathbf{P}=\frac{1}{C_{11}\left(C_{11}+2 C_{12}\right)}\left[\begin{array}{cc}
C_{11}+C_{12} & C_{12} \\
C_{12} & C_{11}+C_{12}
\end{array}\right] .
$$

\section{B. Inductive Coupling}

The magnetic field (inductive) effects in PEEC circuits are modeled using the theory of partial inductances $L p_{i j}$ calculated from the basic expression in (2). In the PEEC method, a partial inductance matrix $\mathbf{L}_{p}$ is constructed with elements of the type $L p_{i j}$. The $\mathbf{L}_{p}$ matrix is usually dense, symmetric, and positive definite for accurate coefficients [14]. The energy using the partial inductance matrix can be written as a quadratic form as

$$
W_{\mathrm{m}}=\frac{1}{2} \boldsymbol{i}^{T} \mathbf{L}_{p} \boldsymbol{i}
$$

where $\boldsymbol{i}$ is the vector of cell currents. Note that in the $\mathbf{L}_{p}$-matrix all elements are positive when calculated using (2). However, if opposing current directions are chosen, coupled inductors can result in negative elements in the $\mathbf{L}_{p}$-matrix.

1) Theoretical Considerations: To perform an analytic stability analysis that includes the partial inductances, a PEEC twocell equivalent circuit is required, so that the problem involves a partial mutual inductance, as shown in Fig. 15. Unfortunately, the quasi-static two-cell is too complicated to yield analytical insight in the configuration in Fig. 15. However, if we discard all the partial mutual coefficients of potential $p_{a b}=0, \forall a, b$ and assume a symmetric geometry two-cell, we have $L p_{12}=L p_{21}$ and $L p_{22}=L p_{11}$. Then, the expression for the input impedance is, by using (21), shown in (26) at the bottom of the page.
The resulting stability condition on the partial mutual inductance $L p_{12}$ (for this simplified symmetric two-cell) is then

$$
\left|L p_{12}\right|<L p_{11}
$$

which is one of the criteria for definite matrices.

Again, for different cell sizes where $L p_{11} \neq L p_{22}$, the stability conditions become too complicated to give any insightful information.

The next section gives an example of a transmission line simulation exploring the properties of the inductance matrix and the corresponding PEEC model solution.

2) Three-Cell Transmission Line: This section considers the stability of a $\left(L_{p}, P, R\right)$ PEEC model for the transmission line in Fig. 16. Note that we chose the different thicknesses of the two transmission line conductors. Each conductor in the transmission line structure is discretized, using projection meshing, into three volume-cells and, therefore, four surface (capacitive) cells. The coefficients of potentials for the parallel surfaces are calculated using closed form formulae [4]. The mutual partial inductances are calculated using two different formulae:

1) LpFilNumT: a thin-tape formula presented in [3] (not taking the thickness of the cells into account which are 25 and $50 \mu \mathrm{m}$, respectively, in this case);

2) LpSumA: a new formulation [21] where the mutual inductance is calculated from a summation of partial selfinductances (calculated using the approach in [14]).

Further, the partial self-inductances are calculated using the scheme in [14].

First, we give the partial inductance matrices using the two different formulations, shown in (28) and (29) at the bottom of the next page. We see some differences in the partial inductance values for the two formulations in the off-diagonal terms: max. $3.5 \%$ relative error in position $(1,6)$. Both the matrices are positive definite which is controlled by checking the determinant of all principal minors to be $>0$, and all the real parts of the eigenvalues of the two $\mathbf{L}$-matrices to be $>0$.

However, when calculating the inverse of the $\mathbf{L}$-matrices, we find some differences to be considerably larger, shown in (30) and (31) on page 30. Both the (inverse) matrices are positive definite, but we notice four positive off-diagonal terms, in the $L_{L p \operatorname{Sum} A}^{-1}$ matrix, representing the couplings $[(1,6),(3,4),(4,3)$, and $(6,1)]$. Since the off-diagonal terms in the inverse of the partial inductance matrix are expected to be negative, we find that the positive elements are a result of inaccurate (mutual) partial inductance values.

Next, we compute the eigenvalues of the 3-cell transmission line PEEC model when using an MNA circuit formulation. The eigenvalues are displayed in Fig. 17 using the two different mutual partial inductance calculation routines.

It is evident from Fig. 17(b) that the eigenvalues differ between the two partial inductance routines where we find a

$$
Z_{\mathrm{IN}}=\frac{\left(s^{4} L p_{11}^{2}+2 L p_{11} P_{22} s^{2}+L p_{11} s^{2} P_{11}+2 L p_{12} P_{22} s^{2}-s^{4} L p_{12}^{2}+P_{22} P_{11}\right) P_{11}}{s\left(L p_{11} s^{2}+P_{11}+L p_{12} s^{2}\right)\left(L p_{11} s^{2}-L p_{12} s^{2}+P_{11}+2 P_{22}\right)} .
$$




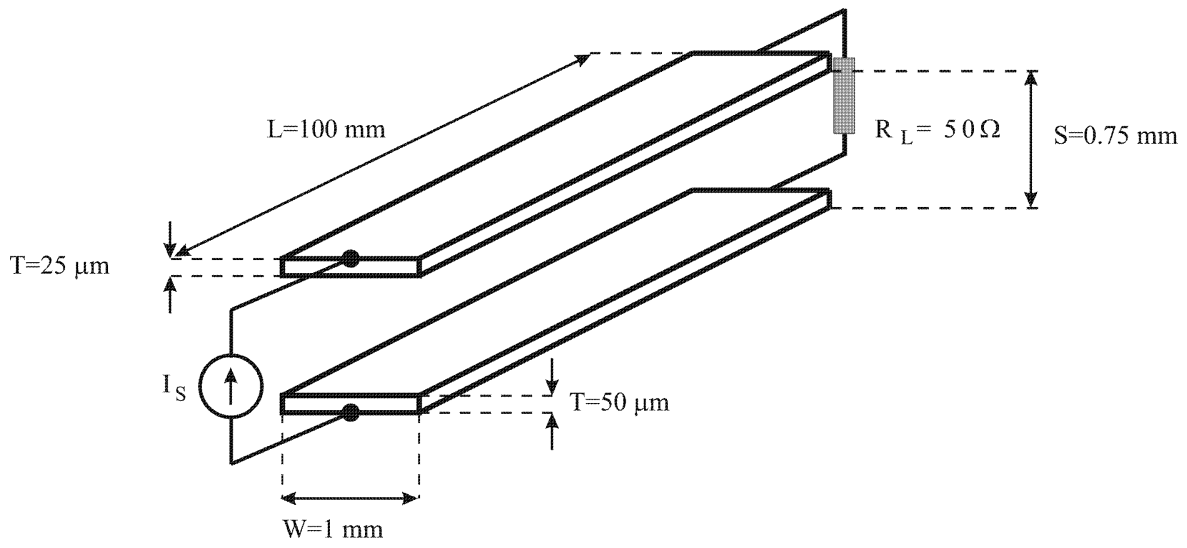

Fig. 16. Transmission line used for numerical experiments.

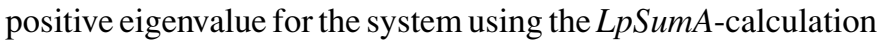
routine. In fact, this is the same routine which produced the positive off-diagonal terms in the inverse partial inductance matrix above. However, for the LpFilNumT-routine, all eigenvalues have negative real parts.

Next, we compare the time-and frequency-domain responses for the transmission line structure. The structure is excited using a 1-mA pulse with a 10-ps rise time (= falltime), a 1.9-ns pulse width. The Backward Euler method is used for the time integration with a 0.5 -ps time step. All the waveforms are stable and very similar, as shown in Fig. 18. However, the system has a positive eigenvalue, shown in Fig. 17, that can cause an unstable response if excited. We can observe a ringing in the input voltage waveform for all the different cases. It is possible to identify the frequency of the ringing waveform (8.925 ns for 25 periods) to $2.80 \mathrm{GHz}$. We continue with the frequencydomain responses for the different cases (Fig. 19). It is possible to make two important observations from the frequency domain simulations:

1) a large peak in the input impedance at $2.8 \mathrm{GHz}$ also observed in the time-domain responses as the $2.8 \mathrm{GHz}$ ringing (Fig. 18);

2) a large peak in the input impedance at $2.7 \mathrm{GHz}$ when using the $\operatorname{LpSumA-calculation}$ routine (Fig. 19).
We conclude that the transmission line PEEC model is stable, for this excitation, for both the calculation routines, even though the MNA system has right half plane eigenvalues and the observed positive elements in the $\mathbf{L}^{-1}$-matrix.

3) Sensitivity Analysis of Mutual Inductance Terms: It is not practical to derive theoretical stability-criteria for the TL, as has previously been done for the one- and two-cell. However, the partial mutual inductance values can be changed in the PEEC-based code to observe the stability for this PEEC model. Fig. 20 shows the time-domain response and the eigenvalues in the complex plane for the pulse-excitation when the mutual terms in the partial inductance matrix (1-6, 6-1,3-4, 4-3) are reduced by $30 \%$ of the self-term (1-1), and the mutual terms are calculated using the LpFilNumT-routine. Positive eigenvalues are found $\left(1.4210^{-7}\right)$, as are positive off-diagonal terms, in the inverse of the partial inductance matrix. Again, the $\mathbf{L}_{p}$-matrix and its inverse are positive definite, as shown in the previous section.

By further altering the mutual terms, now to $32.95 \%$ of the self-term, an unstable system is obtained. Fig. 21 shows the response and location of poles in the complex plane for this test. We see from this model simulation that by altering some partial mutual inductance terms by a few percentages, an unstable system can result with an eigenvalue of very large magnitude.

$$
\begin{aligned}
\mathbf{L}_{L p \text { FilNum } T}= & {\left[\begin{array}{cccccc}
31.0631 & 4.5878 & 1.7441 & 22.6547 & 4.5371 & 1.7440 \\
4.5878 & 31.0631 & 4.5878 & 4.5371 & 22.6547 & 4.5371 \\
1.7441 & 4.5878 & 31.0631 & 1.7440 & 4.5371 & 22.6547 \\
22.6547 & 4.5371 & 1.7440 & 31.2274 & 4.5878 & 1.7441 \\
4.5371 & 22.6547 & 4.5371 & 4.5878 & 31.2274 & 4.5878 \\
1.7440 & 4.5371 & 22.6547 & 1.7441 & 4.5878 & 31.2274
\end{array}\right] \quad[\mathrm{nH}] } \\
\mathbf{L}_{L p \operatorname{Sum} A}= & {\left[\begin{array}{cccccc}
31.0631 & 4.5877 & 1.7464 & 22.7446 & 4.5382 & 1.6826 \\
4.5877 & 31.0631 & 4.5877 & 4.5382 & 22.7446 & 4.5382 \\
1.7464 & 4.5877 & 31.0631 & 1.6826 & 4.5382 & 22.7446 \\
22.7446 & 4.5382 & 1.6826 & 31.2274 & 4.5878 & 1.7413 \\
4.5382 & 22.7446 & 4.5382 & 4.5878 & 31.2274 & 4.5878 \\
1.6826 & 4.5382 & 22.7446 & 1.7413 & 4.5878 & 31.2274
\end{array}\right][\mathrm{nH}] . }
\end{aligned}
$$




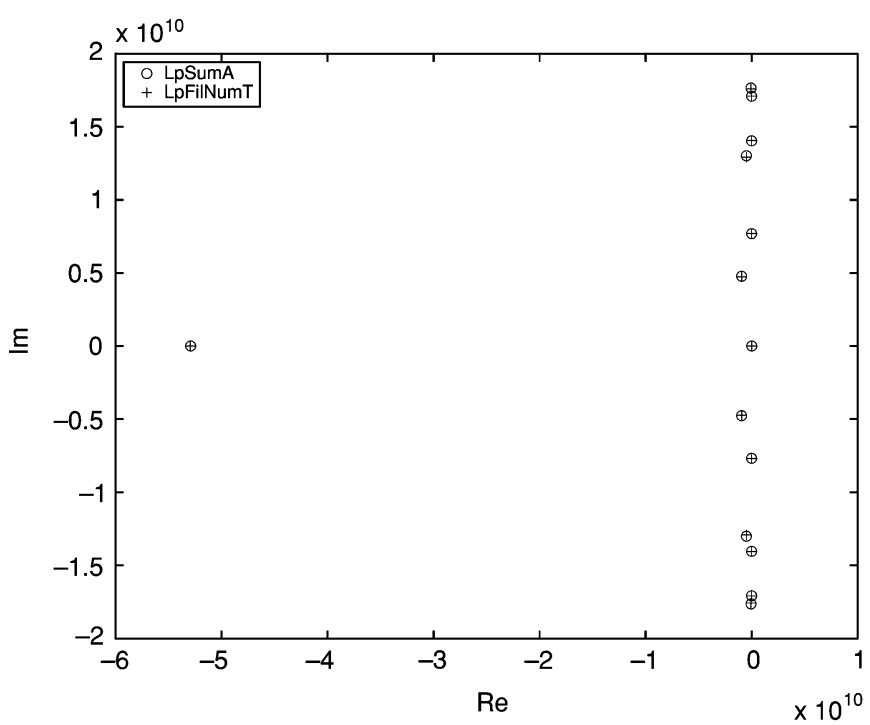

(a)

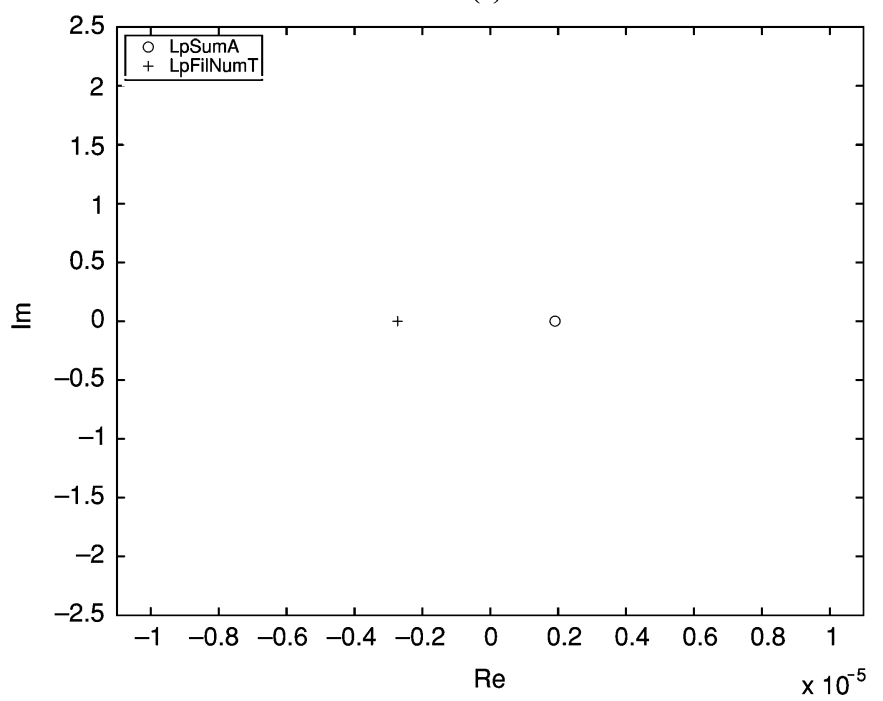

(b)

Fig. 17. Eigenvalues for MNA based system matrix. (a) Global view. (b) Near origin.
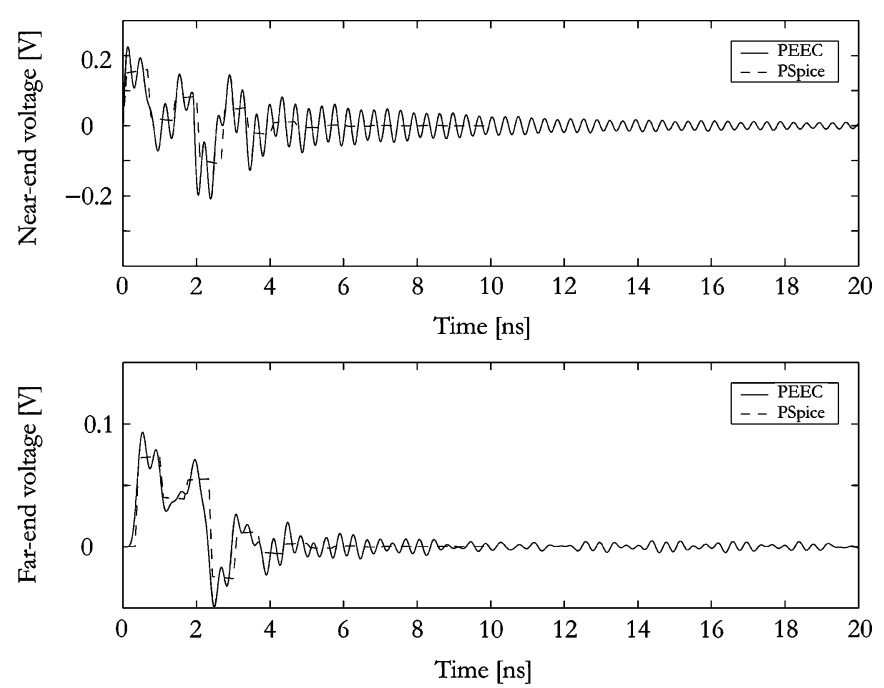

Fig. 18. Transmission line time-domain response for the NA method using LpSumA-routine. Comparison between PEEC and PSpice transmission line (T) element.
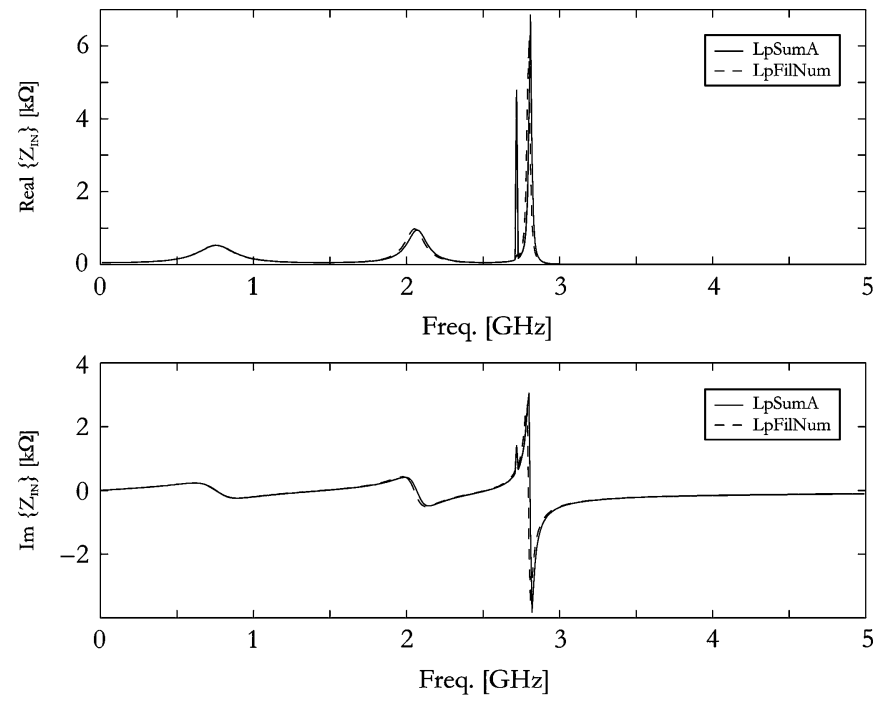

Fig. 19. Input impedance for the TL in Fig. 16.

$$
\mathbf{L}_{L p \text { FilNum } T}^{-1}=\left[\begin{array}{cccccc}
6.866 & -0.195 & -0.036 & -4.931 & -0.121 & -0.036 \\
-0.195 & 6.891 & -0.195 & -0.121 & -4.907 & -0.121 \\
-0.036 & -0.195 & 6.866 & -0.036 & -0.121 & -4.931 \\
-4.931 & -0.121 & -0.036 & 6.829 & -0.189 & -0.035 \\
-0.121 & -4.907 & -0.121 & -0.189 & 6.853 & -0.189 \\
-0.036 & -0.121 & -4.931 & -0.035 & -0.189 & 6.829
\end{array}\right] \cdot 10^{7}\left[\mathrm{H}^{-1}\right]
$$

and

$$
\mathbf{L}_{L p \operatorname{Sum} A}^{-1}=\left[\begin{array}{cccccc}
6.928 & -0.194 & -0.079 & -4.996 & -0.121 & 0.009 \\
-0.194 & 6.952 & -0.194 & -0.121 & -4.972 & -0.121 \\
-0.079 & -0.194 & 6.928 & 0.009 & -0.121 & -4.996 \\
-4.996 & -0.121 & 0.009 & 6.890 & -0.188 & -0.076 \\
-0.121 & -4.972 & -0.121 & -0.188 & 6.914 & -0.188 \\
0.009 & -0.121 & -4.996 & -0.076 & -0.188 & 6.890
\end{array}\right] \cdot 10^{7}\left[\mathrm{H}^{-1}\right]
$$



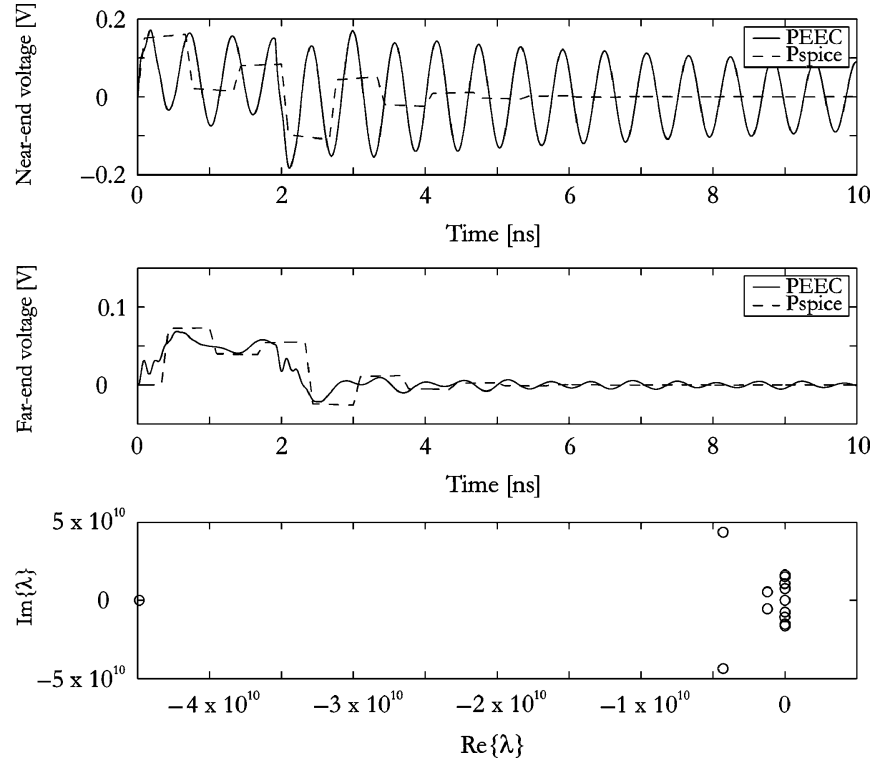

Fig. 20. TL response using PEEC and PSpice transmission line (T) element and eigenvalues for PEEC for inaccurate partial mutual inductance values.
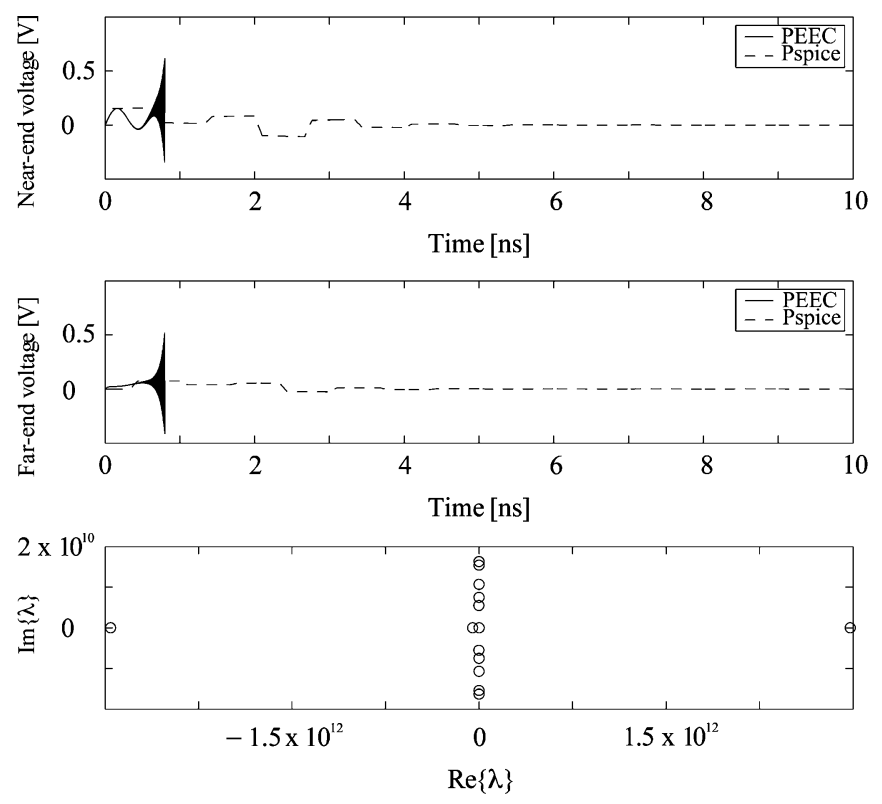

Fig. 21. TL response using PEEC and PSpice transmission line (T) element and eigenvalues for PEEC for inaccurate partial mutual inductance values.

However, for this case, both the $L_{p}$-matrix and its inverse are indefinite.

We conclude the following.

1) The manual alteration of the partial mutual inductance is fully comparable with the previously observed errors in the analytical and numerical integration routines, causing an unstable model.

2) Seemingly stable systems can have positive definite partial inductance matrices $\left(L_{p}\right.$ and $\left.L_{p}^{-1}\right)$ and small positive eigenvalues.
3) For clearly unstable systems, we observed negative definite partial inductance matrices and large positive eigenvalues, as expected.

\section{CONCLUSION}

This paper clearly shows that accuracy and even stability issues exist for lossless quasi-static $\left(L_{P}, P\right)$ PEEC models which can be treated by an ordinary differential equation solver. Many of these issues are necessary conditions for the stability also of full-wave $\left(L_{P}, P, \tau\right)$ PEEC models.

The impact of inaccurate partial elements are shown theoretically and experimentally. We have shown that poorly calculated partial elements can shift eigenvalues for the complete PEEC system into the right half plane, thus causing instability. It has also been shown that poor meshing may result in negative capacitance values as a possible source of instabilities. The paper gives references to suitable partial element calculation routines and suggests a meshing strategy, called projection meshing, to overcome sources of inaccurate partial element values, thus improving PEEC model stability.

\section{OBSERVATION}

For full-wave PEEC models applied to small geometries where a maximum dimension $d<\left(\lambda_{\min } / 10\right)$ is similar to quasistatic models, we observe that the theory presented in this paper clearly applies to full-wave models in the $\lim _{d \rightarrow 0}$.

\section{REFERENCES}

[1] V. Okhmatovski, A. Cangellaris, and J. Morsey, "Accuracy-related issues in electronic modeling of high-speed interconnects," in Proc. IEEE Int. Symp. EMC, Boston, MA, 2003, pp. 338-346.

[2] ITRS. (2003) International Technology Roadmap for Semiconductors 2003 Edition [Online]. Available: http://public.itrs.net/Files/2003ITRS/ Home2003.htm

[3] A. E. Ruehli, "Inductance calculations in a complex integrated circuit environment," IBM J. Res. Develop., vol. 16, no. 5, pp. 470-481, Sep. 1972.

[4] A. E. Ruehli and P. A. Brennan, "Efficient capacitance calculations for three-dimensional multiconductor systems," IEEE Trans. Microw. Theory Tech., vol. 21, no. 2, pp. 76-82, Feb. 1973.

[5] A. E. Ruehli, "Equivalent circuit models for three dimensional multiconductor systems," IEEE Trans. Microw. Theory Tech., vol. MTT-22, no. 3, pp. 216-221, Mar. 1974.

[6] C. Ho, A. Ruehli, and P. Brennan, "The modified nodal approach to network analysis," IEEE Trans. Circuits Syst., vol. CS-22, no. 6, pp. 504 509, Jun. 1975.

[7] A. E. Ruehli, G. Antonini, J. Esch, J. Ekman, A. Orlandi, and A. Mayo, "Nonorthogonal PEEC formulation for time and frequency-domain EM and circuit modeling," IEEE Trans. Electromagn. Compat., vol. 45, no. 2, pp. 167-176, May 2003.

[8] B. Rynne, "Comments on a stable procedure in calculating the transient scattering by conducting surfaces of arbitary shape," IEEE Trans. Antennas Propag., vol. 41, no. 4, pp. 517-520, Apr. 1993.

[9] J. Ekman, G. Antonini, A. Orlandi, and A. E. Ruehli, "Stability of PEEC models with respect to partial element accuracy," in Proc. IEEE Int. Symp. EMC, Santa Clara, CA, 2004, pp. 271-276.

[10] A. E. Ruehli, P. A. Brennan, and H. W. Young, "Recent progress in capacitance computation methods," in Proc. IEEE Int. Symp. Circuits and Systems, Phoenix, AZ, 1975, pp. 135-138.

[11] B. Krauter and L. T. Pileggi, "Generating sparse partial inductance matrices with guaranteed stability," in Proc. Int. Conf. Computer Aided Design, San Jose, CA, 1995, pp. 45-52. 
[12] M. Beattie and L. Pileggi, "Efficient inductance extraction via windowing," in Proc. Design, Automation and Test in Europe, Munich, Germany, 2001, pp. 430-436.

[13] G. Zhong, C.-K. Koh, and K. Roy, "On-chip interconnect modeling by wire duplication," in Proc. Int. Conf. Computer Aided Design, San Jose, CA, 2002, pp. 341-346.

[14] P. K. Wolff and A. E. Ruehli, "Inductance computations for complex three dimensional geometries," in Proc. IEEE Int. Symp. Circuits and Systems, New York, 1981, pp. 16-19.

[15] A. E. Ruehli, U. Miekkala, A. Bellen, and H. Heeb, "Stable time domain solutions for EMC problems using PEEC circuit models," in Proc. IEEE Int. Symp. EMC, Chicago, IL, 1994, pp. 371-376.

[16] A. Bellen, N. Guglielmi, and A. Ruehli, "Methods for linear systems of circuit delay differential equations of neutral type," IEEE Trans. Circuits Syst., vol. 46, no. 1, pp. 212-216, Jan. 1999.

[17] A. Cangellaris, W. Pinello, and A. E. Ruehli, "Stabilization of time domain solutions of EFIE based on partial element equivalent circuit models," in Proc. IEEE Antennas and Propagation Society Int. Symp., Montreal, QC, Canada, 1997, pp. 966-969.

[18] H. Kim and C. C.-P. Chen, "Be Careful of Self and Mutual Inductance Formulae," VLSI-EDA LAB, Univ. Wisconsin, Madison, Tech. Rep., 1999.

[19] G. Antonini and A. E. Ruehli, "Fast multipole and multifunction PEEC methods," IEEE Trans. Mobile Comput., vol. 2, no. 4, pp. 288-298, Oct.Dec. 2003.

[20] W. Cheney and D. Kincaid, Numerical Analysis, 2nd ed. Pacific Grove, CA: Brooks/Cole, 1996.

[21] G. Zhong and C.-K. Koh, "Exact closed form formula for partial mutual inductances of on-chip interconnects," in Proc. 2002 Int. Conf. Computer Design, Freiburg, Germany, 2002, pp. 428-433.

[22] G. Antonini, A. Orlandi, and A. E. Ruehli, "Analytical integration of quasi-static potential intergrals on nonorthogonal coplanar quadrilaterals for the PEEC method," IEEE Trans. Electromagn. Compat., vol. 44, no. 2 , pp. 399-403, May 2002.

[23] G. Antonini, J. Ekman, and A. Orlandi, "3-D PEEC capacitance calculations," in Proc. IEEE Int. Symp. EMC, Boston, MA, 2003, pp. 630-635.

[24] Z. Bai, P. M. Dewilde, and R. W. Freund, "Reduced-Order Modeling," Bell labs., Tech. Rep., 2002, Numerical Analysis Manuscript No. 02-4-13.

[25] (2003). PSpice A/D Datasheet [Online]. Available: http://www.orcad. com/pdf/PSpiceAD_DS.pdf

[26] G. Antonini, J. Ekman, and A. Orlandi, "Full wave time domain PEEC formulation using a modified nodal analysis approach," in Proc. EMC Europe, Eindhoven, The Netherlands, 2004, pp. 12-17.

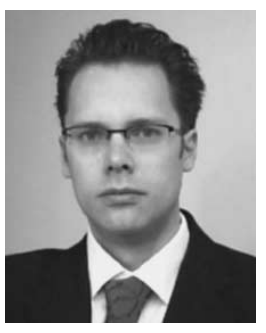

Jonas Ekman (M'03) was born in Boden, Sweden, in 1972. He received the Ph.D. degree in electrical engineering from Lule University of Technology, Luleå Sweden, in 2003.

Since 2003, he has been working as a Researcher at EISLAB, Luleå University of Technology. He is currently at University of L'Aquila EMC Laboratory, L'Aquila, Italy, working on full-wave, time domain, partial element equivalent circuit (PEEC) modeling with financial support from The Swedish Research Council. His field of study is computational electromagnetics and, in particular, the use of the PEEC method for realistic electromagnetic modeling.

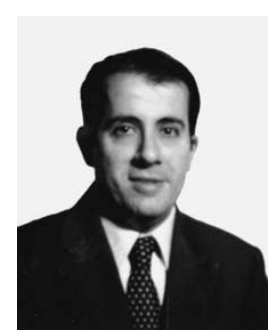

Giulio Antonini (M'96-SM'05) received the Laurea degree (summa cum laude) from the University of L'Aquila, L'Aquila, Italy, and the Ph.D. degree from the University of Rome "La Sapienza," Rome, Italy, in 1994 and 1998, respectively, both in electrical engineering.

Since 1998, he has been with the UAq EMC Laboratory, Department of Electrical Engineering, University of L'Aquila, where he is a tenured Senior Researcher. Since 1998, he has collaborated with the IBM T. J. Watson Research Center, Yorktown Heights, NY, in the development of algorithms for PEEC modeling. He has authored or coauthored more than 100 technical papers and given seven keynote lectures at international conferences. He holds one European patent. His research interests focus on EMC analysis, numerical modeling, and signal integrity for high-speed digital systems.

Dr. Antonini received the IEEE TRANSACTIONS ON ELECTROMAGNETIC CompatibiLity Best Paper Award in 1997, the CST University Publication Award in 2004, and the IBM Shared University Research Award in 2004 and 2005. He is a member of the TC-9 Committee of the IEEE EMC Society and Secretary of the TC-10 Committee of the same IEEE Society. He is in the Technical Program Committee of the Date-06 Conference and serves as a Reviewer for a number of IEEE journals.

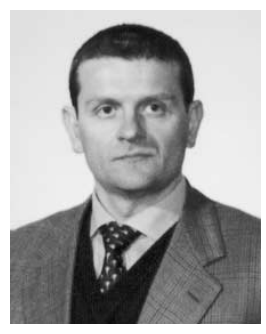

Antonio Orlandi (M'90-SM'97) was born in Milan, Italy, in 1963. He received the Laurea degree in electrical engineering from the University of Rome "La Sapienza," Rome, Italy, in 1988.

From 1988 to 1990 , he was with the Department of Electrical Engineering, University of Rome "La Sapienza." Since 1990, he has been with the Department of Electrical Engineering, University of L'Aquila, L'Aquila, Italy, where he is currently Full Professor at the UAq EMC Laboratory. He is the author of more than 150 technical papers. He has published in the field of electromagnetic compatibility in lightning protection systems and power drive systems. His current research interests are numerical methods and modeling techniques to approach signal/power integrity and EMC/EMI issues in high-speed digital systems.

Dr. Orlandi received the IEEE TRANSACTIONS ON ELECTROMAGNETIC CompatiBiLity Best Paper Award in 1997, the IEEE EMC Society Technical Achievement Award in 2003, the IBM Shared University Research Award in 2004, and the CST University Award in 2004. He is member of the Education, TC-9 Computational Electromagnetics, and Vice-Chairman of the TC-10 Signal Integrity Committees of the IEEE EMC Society, and Chairman of the "EMC Innovation" Technical Committee of the International Zurich Symposium and Technical Exhibition on EMC. From 1996 to 2000, he was Associate Editor of the IEEE TRANSACTIONS ON ELECTROMAGNETIC COMPATIBILITY and now serves as Associate Editor of the IEEE TRANSACTIONS ON MOBILE COMPUTING.

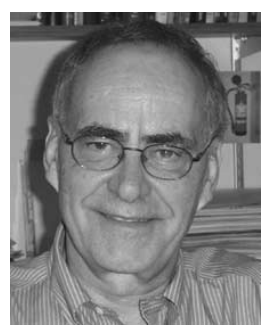

Albert E. Ruehli (M'65-SM'74-F'84-LF'03) received the Ph.D. degree in electrical engineering from the University of Vermont, Burlington, in 1972.

He has been a member of various projects with IBM, Yorktown Heights, NY, including mathematical analysis, semiconductor circuits and devices modeling, and as manager of a VLSI design and CAD group. Since 1972, he has been at the IBM T.J. Watson Research Center, Yorktown Heights, where he now is a Research Staff Member in the Electromagnetic Analysis Group. He is an author or coauthor of over 100 technical papers, and he is the editor of Circuit Analysis, Simulation and Design, parts 1 and 2 (New York: North-Holland 1986, 1987).

Dr. Ruehli has served in numerous capacities for the IEEE. He was the Technical and General Chairman of the ICCD International Conference in 1984 and 1985 , respectively. He has been a member of the IEEE ADCOM for the Circuits and Systems Society and an Associate Editor for the IEEE TRANSACTION ON COMPUTER-AIDED DESIGN. He has given talks at universities including keynote addresses and tutorials at conferences and has organized many sessions. $\mathrm{He}$ received IBM Research Division or IBM Outstanding Contribution Awards in 1975, 1978, 1982, 1995, and 2000. In 1982, he received the Guillemin-Cauer Prize Award for his work on waveform relaxation, and in 1999, he received a Golden Jubilee Medal, both from the IEEE CAS Society. In 2001, he received a Certificate of Achievement from the IEEE EMC Society for inductance concepts and the partial element equivalent circuit (PEEC) method. He received the 2005 Richard R. Stoddart Award from the IEEE EMC Society for outstanding technical performance. He is also a member of SIAM. 